\title{
Caracterización clínico epidemiológica de pacientes hospitalizados con diagnóstico de falla cardiaca descompensada con fracción de eyección reducida
}

\author{
Epidemiological and clinical characterization of \\ patients hospitalized for decompensated heart \\ failure with reduced ejection fraction
}

\author{
Laura Catalina Arcos-Medina, Arnold Méndez-Toro, Ingrid Tatiana Rojas-Ruiz, \\ Sergio Guillermo Torres-Riveros, Sebastian Camilo Tabares-Rodríguez • \\ BogotÁ, D.C. (Colombia)
}

DOI: https://doi.org/10.36104/amc.2020.1233

\section{Resumen}

Objetivo: describir las características clínicas, bioquímicas y tratamiento de pacientes con falla cardiaca descompensada con fracción de eyección reducida hospitalizados en el año 2015 en el Hospital Militar Central de Bogotá.

Metodología: estudio descriptivo en el que se revisaron registros clínicos de pacientes mayores de 18 años hospitalizados por falla cardiaca descompensada con fracción de eyección reducida en el año 2015. Se registraron variables clínicas, bioquímicas, ecocardiográficas y de tratamiento. Se realizó análisis univariado, reportando para variables cualitativas proporciones, para variables cuantitativas medidas de tendencia central y para variables que no siguen distribución normal, medianas y cuartiles 1 y 3 .

Resultados: se analizaron registros de 114 pacientes, con edad promedio de 74.8 años, $69.3 \%$ hombres. La etiología de la falla cardiaca fue hipertensiva $66.7 \%$ e isquémica $60.5 \%$, la no adherencia al tratamiento fue el principal factor precipitante de descompensación, se encontró alta adherencia a guías de manejo al egreso con medicación en dosis subóptimas, y titulación ambulatoria en 32\% de los pacientes, el 38\% reingresaron al menos una vez dentro de los primeros 30 días. Un $25.4 \%$ requirió manejo en UCI con mortalidad de $18 \%$ y para reingresos tempranos de $16 \%$, siendo de $3 \%$ para pacientes con ingresos tardíos.

Conclusión: en este estudio el perfil clínico es semejante a los reportados en otras series. La adherencia a guías de manejo es adecuada pero en dosis subóptimas. Existe un alto porcentaje de reingreso registrados y de mortalidad intrahospitalaria. (Acta Med Colomb 2020; 45. DOI: https:// doi.org/10.36104/amc.2020.1233).

Palabras clave: falla cardiaca, readmisión de pacientes, mortalidad

\footnotetext{
Abstract

Objective: to describe the clinical and biochemical characteristics and treatment of patients with decompensated heart failure with reduced ejection fraction hospitalized during 2015 at the Hospital Militar Central in Bogotá.

Methods: a descriptive study reviewing the clinical records of patients over the age of 18 who were hospitalized due to decompensated heart failure with reduced ejection fraction during 2015. Clinical, biochemical, echocardiographic and treatment variables were recorded. A univariate analysis was performed reporting percentages for qualitative variables, measures of central tendency for quantitative variables, and medians and first and third quartiles for variables with a non-normal distribution.
}

Dra. Laura Catalina Arcos-Medina: Especialista en Medicina Interna, Cardiología y Ecocardiografía, Hospital Militar Central, Hospital Universitario Mayor - Mederi; Dr. Arnold Méndez-Toro: Especialista en Medicina Interna y Cardiología, Hospital Universitario Nacional de Colombia; Ingrid Tatiana RojasRuiz: Enfermera, Especialista en Calidad en Salud y Epidemiología, MsC Epidemiología, Profesor Auxiliar Universidad Nacional de Colombia, Facultad de Enfermería; Sergio Guillermo Torres-Riveros y Sebastián Camilo Tabares-Rodríguez: Estudiantes de Medicina Universidad Militar Nueva Granada. Bogotá, D.C. (Colombia).

Correspondencia: Ingrid Tatiana Rojas-Ruiz (Bogotá, D.C. Colombia).

E-mail: itrojasr@unal.edu.co

Recibido: 28/VI/2018 Aceptado: 29/I/2020 
Results: the medical records of 114 patients were analyzed (average age 74.8 years; $69.3 \%$ males). The etiology of the heart failure was hypertension in $66.7 \%$ and ischemia in $60.5 \%$. Noncompliance was the main precipitating factor for decompensation. High adherence to management guidelines was seen on discharge, with medications at suboptimal doses and ambulatory titration in $32 \%$ of patients; $38 \%$ were readmitted at least once during the first 30 days. Altogether, $25.4 \%$ required ICU care, with a mortality rate of $18 \%$; there was a $16 \%$ mortality rate for early readmissions and $3 \%$ for patients with late admissions.

Conclusion: the clinical profile in this study is similar to what is reported in other research. Adherence to management guidelines is adequate, but at suboptimal doses. There is a high percentage of recorded readmissions and hospital mortality. (Acta Med Colomb 2020; 45. DOI: https:// doi.org/10.36104/amc.2020.1233).

Key words: heart failure, patient readmission, mortality

\section{Introducción}

La insuficiencia cardiaca constituye una enfermedad con alta prevalencia y se espera un aumento en su incidencia y de un incremento en los factores de riesgo cardiovascular (1-2). En Colombia, la enfermedad cardiovascular continua como la primera causa de muerte, reportándose en 136 por cien mil habitantes, siendo la falla cardiaca uno de sus principales determinantes. Se estima que existen 1.1 millones de pacientes con diagnóstico de falla cardiaca con una prevalencia de 2.3\% para Latinoamérica (3). En los años 2009 al 2012 (4) aumentó el ingreso a urgencias de pacientes con falla cardiaca descompensada en $121 \%, 10 \%$ en consulta externa y $43 \%$ en pacientes hospitalizados, lo cual refleja el envejecimiento de la población, incrementando el consumo de recursos del sistema como lo demuestran las estadísticas del Ministerio de Salud y Protección Social.

Adicionalmente existen publicaciones nacionales, en las cuales se reporta un uso bajo de medicamentos como betabloqueadores, inhibidores de la enzima convertidora de angiotensina y bloqueadores de los receptores de angiotensina II (5), un seguimiento pobre de los pacientes que están en terapia con dispositivos como el resincronizador y el desfibrilador implantable y un porcentaje de reingresos hospitalarios anuales de $40.8 \%$ en una población de pacientes atendidos en un hospital general, con un costo promedio de 6427887 pesos colombianos por hospitalización (6).

Las recomendaciones para el cuidado del paciente con falla cardiaca se encuentran establecidas y documentadas en la literatura e incluyen la terapia farmacológica, cambios de estilo de vida, manejo intrahospitalario, seguimiento ambulatorio y cuidado paliativo (7-10). Sin embargo, a pesar de estas directrices ampliamente disponibles para el personal de la salud, los índices de hospitalización siguen siendo altos; por lo tanto, con el fin de prevenir exacerbación de los síntomas, hospitalizaciones y reingresos, se debe establecer las deficiencias de atención y necesidades e involucrar al proveedor de atención directa como un actor principal en su evaluación convirtiéndose en una necesidad contar con programas de falla cardiaca que mantengan la continuidad de las medidas de intervención más allá del alta hospitalaria o para evitar el ingreso a hospitalización o cuidado intensivo.

En el Hospital Militar Central no hay estadísticas contundentes sobre la prevalencia de la enfermedad, el seguimiento clínico, ni la optimización del manejo, así como los desenlaces de estos pacientes, sin embargo, el diagnóstico de falla cardiaca sigue encontrándose dentro de las 10 principales causas de hospitalización.

Por esta razón, se considera prioritario la caracterización y el conocimiento de este grupo de población con alta tasa de morbilidad, mortalidad y costos al sistema, en aras de plantear un programa de seguimiento estructurado como una Clínica de Falla Cardiaca en el HMC respondiendo a las necesidades de la población, en búsqueda de estándares de calidad y eficiencia ya que el pronóstico puede verse sustancialmente modificado mediante intervenciones médicas, uso de dispositivos y trasplante de corazón, disminuyendo costos en la atención, porcentajes de hospitalización e impactando tanto en mortalidad como en morbilidad.

\section{Metodología}

Se trata de un estudio descriptivo de cohorte retrospectiva en pacientes con insuficiencia cardiaca descompensada con FEVI reducida que son admitidos a un hospital de cuarto nivel de las fuerzas militares de Colombia, durante un periodo de seguimiento de un año.

Los criterios de inclusión fueron adultos mayores de 18 años con diagnóstico de insuficiencia cardiaca descompensada con fracción de eyección menor a 40\%, los criterios de exclusión: pacientes con diagnóstico de insuficiencia cardiaca con fracción de eyección mayor de $40 \%$ o menor de $40 \%$ con falla cardiaca no descompensada.

Se tomó el total de registros de pacientes con los códigos CIE 10 correspondientes al diagnóstico de insuficiencia cardiaca I500-I509 entre el $1^{\circ}$ de enero y 31 de diciembre de 2015. Se encontraron registros de 459 pacientes con estos diagnósticos, se realizó revisión de cada historia clínica para determinar si el código diligenciado correspondía al diagnóstico clínico de falla cardiaca y si sus ingresos eran a causa de 
descompensación de la misma, igualmente se seleccionaron los ecocardiogramas transtorácicos y transesófagicos de cada uno de los pacientes para confirmar el criterio de fracción de eyección reducida, un total de 114 pacientes cumplieron con los criterios de inclusión.

Se diseñó un formato para la recolección de la información que contenía los datos de identificación, fecha de ingreso, egreso y las características clínicas (etiología de la enfermedad, comorbilidades, factores precipitantes, examen físico al ingreso) paraclínicos, hallazgos ecocardiográficos, días de hospitalización, días de UCI, muerte y optimización ambulatoria.

Para medir el desenlace muerte se revisaron todas las historias clínicas, y para aquellos pacientes que durante el 2016 no se encontraron registros de atención, se realizó llamada telefónica para confirmar su supervivencia durante el periodo de seguimiento.

\section{Resultados}

Se realizó una búsqueda en el sistema de historias clínicas del HMC de los pacientes hospitalizados en el año 2015, quienes entre sus códigos CIE10 presentara el diagnóstico de insuficiencia cardiaca (I500 - I501), obteniéndose 509 pacientes. Se verificó que dicho código correspondiera al diagnóstico de falla cardiaca en 459 pacientes. En este grupo se revisaron los ecocardiogramas encontrándose una FEVI reducida (definida como $\leq 40 \%$ ) en $28.3 \%$ que corresponde a 130 pacientes. En esta población con FEVIr se encontró al menos una admisión por falla cardiaca descompensada en 114 pacientes para el año 2015 en los cuales se realizó el análisis (Figura 1).

\section{Características sociodemográficas, clínicas y comorbilidades (Tabla 1)}

El $62 \%$ de los pacientes tenían fracción de eyección $<30 \%$, y $38 \%$, FE $<40 \%$, la edad media de la muestra fue 74.8 años, el $42 \%$ de la población tenía edad igual o mayor a 80 años, el $69.3 \%$ eran hombres. La etiología de la falla cardiaca fue hipertensiva en $66.7 \%$, isquémica $60.5 \%$, valvular $20.2 \%$, otro factor etiológico fue reportado en $8.8 \%$ de los casos; la no adherencia al tratamiento fue el principal precipitante con $50 \%$ de los casos, seguido de un factor infeccioso en $28.1 \%$ de los casos y síndrome coronario agudo en $21.9 \%$. Presentaron una comorbilidad

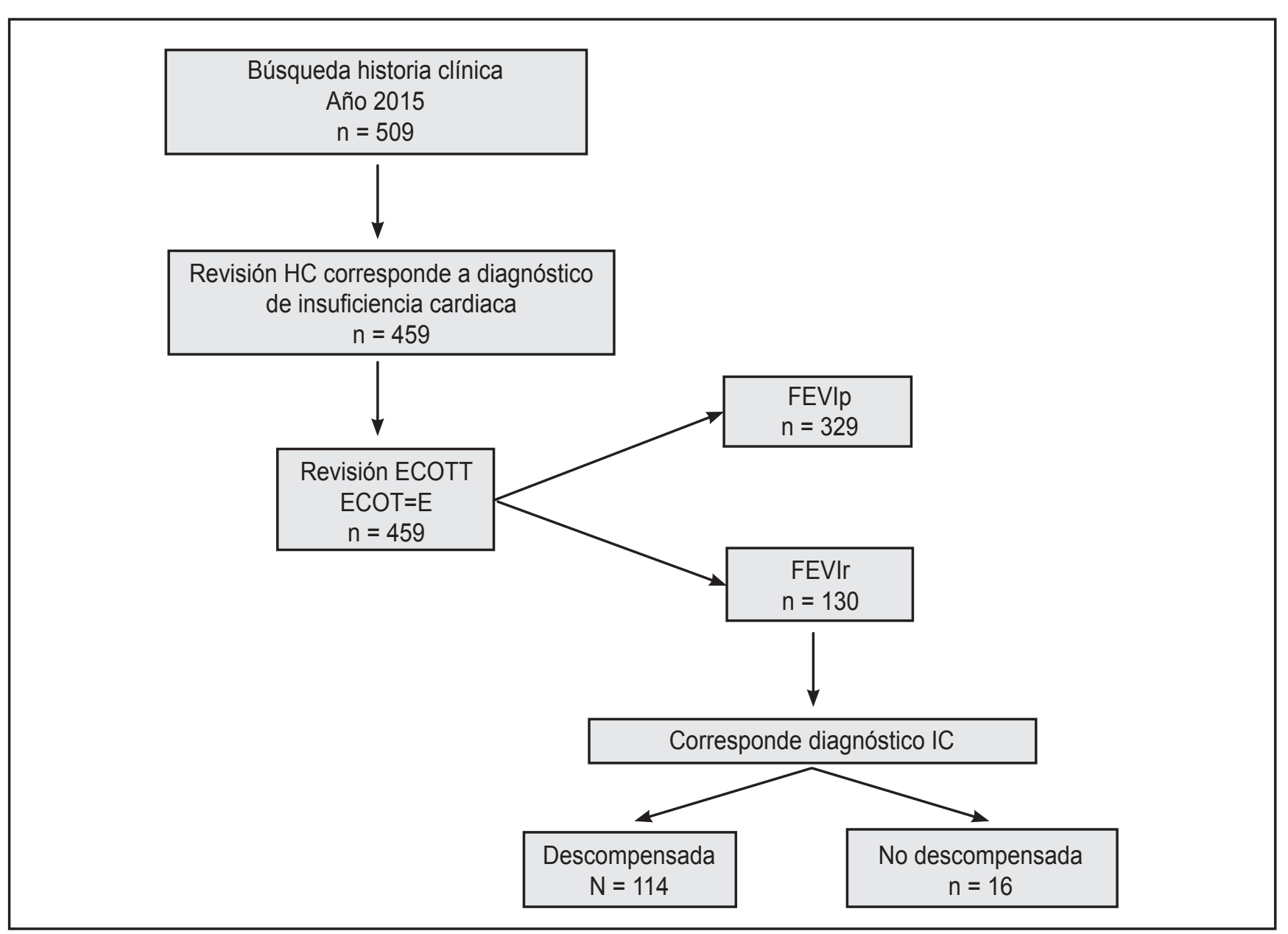

Figura 1. Selección de los registros clínicos. (Selección de los pacientes; HC, historia clínica; ECOTT, ecocardiograma transtorácico, ECOT-E, ecocardiograma transesofágico, FEVIp/r, fracción de eyección del ventrículo izquierdo preservadal reducida, IC insuficiencia cardiaca). 
Tabla 1. Características sociodemográficas, clínicas y comorbilidades.

\begin{tabular}{|c|c|c|}
\hline & n & $\%$ \\
\hline Edad & 74.8 & 11.8 \\
\hline Género (masculino) & 79 & 69.3 \\
\hline \multicolumn{3}{|l|}{ Etiología } \\
\hline Hipertensiva & 76 & 66.7 \\
\hline Isquémica & 69 & 60.5 \\
\hline Valvular & 23 & 20.2 \\
\hline Otra & 10 & 8.8 \\
\hline Chagas & 8 & 7 \\
\hline Idiopática & 3 & 2.6 \\
\hline Taquicardiomiopatía & 4 & 3.5 \\
\hline \multicolumn{3}{|l|}{ Precipitante } \\
\hline No adherencia & 57 & 50 \\
\hline Infeccioso & 32 & 28.1 \\
\hline SCA & 25 & 21.9 \\
\hline Arritmia & 20 & 17.5 \\
\hline Valvular & 11 & 96 \\
\hline \multicolumn{3}{|l|}{ Comorbilidad } \\
\hline HTA & 105 & 92.1 \\
\hline Enfermedad coronaria & 70 & 61.4 \\
\hline ERC & 60 & 52.6 \\
\hline Hipo/hipertiroidismo & 56 & 49.1 \\
\hline EPOC & 52 & 45.6 \\
\hline HTP & 49 & 43 \\
\hline DM2 & 34 & 29.8 \\
\hline FA & 31 & 27.2 \\
\hline DL & 25 & 21.9 \\
\hline TVP & 15 & 13.2 \\
\hline Cáncer & 10 & 8.8 \\
\hline Hepatopatía & 9 & 7.9 \\
\hline $\mathrm{ACV} / \mathrm{AIT}$ & 8 & 7 \\
\hline TEP & 3 & 2.6 \\
\hline SAHOS & 3 & 3.6 \\
\hline \multicolumn{3}{|l|}{ Stevenson } \\
\hline A & 10 & 8.8 \\
\hline B & 92 & 80.6 \\
\hline $\mathrm{C}$ & 10 & 8.8 \\
\hline $\mathrm{L}$ & 2 & 1.8 \\
\hline \multicolumn{3}{|l|}{ NYHA } \\
\hline II & 11 & 9.6 \\
\hline III & 55 & 48.2 \\
\hline IV & 47 & 41.2 \\
\hline \multicolumn{3}{|l|}{ Estadio } \\
\hline B & 2 & 1.8 \\
\hline $\mathrm{C}$ & 93 & 81.6 \\
\hline $\mathrm{D}$ & 17 & 14.9 \\
\hline \multicolumn{3}{|l|}{ Examen físico } \\
\hline TAS promedio $\mathrm{mmHg}$ & 131.8 & 24.7 \\
\hline $\mathrm{TAD}$ promedio $\mathrm{mmHg}$ & 76.6 & 16.8 \\
\hline $\mathrm{FC} * 1 \mathrm{pm}$ & & \\
\hline \multicolumn{3}{|l|}{ Inicio } \\
\hline Crónica descompensada & 99 & 86.8 \\
\hline De novo & 9 & 7.9 \\
\hline \multicolumn{3}{|l|}{ Número hospitalizaciones } \\
\hline Una & 71 & 62.4 \\
\hline Dos & 27 & 23.7 \\
\hline Tres & 11 & 9.6 \\
\hline Cuatro & 2 & 1.7 \\
\hline Cinco & 2 & 1.7 \\
\hline Siete & 1 & 0.9 \\
\hline \multicolumn{3}{|c|}{$\begin{array}{l}\text { SCA, síndrome coronario agudo. HTA, hipertensión arterial. ERC, enfermedad renal } \\
\text { crónica. EPOC, enfermedad pulmonar obstructiva crónica. http, hipertensión pul- } \\
\text { monar. FA, fibrilación auricular. DL, dislipidemia.TVP, trombosis venosa profunda. } \\
\text { ACVIAIT, accidente cerebrovascular / accidente isquémico transitorio. TEP, trom- } \\
\text { boembolismo pulmonar. SAHOS, Síndrome de apneal hipopnea obstructiva del sueño } \\
\text { * Datos reportados como mediana y rango IQ.TAS, tensión arterial sistólica.TAD, } \\
\text { tensión arterial diastólica.FC, frecuencia cardiaca }\end{array}$} \\
\hline
\end{tabular}

significativa: el $92.1 \%$ de los pacientes con hipertensión arterial, el $61.4 \%$ con enfermedad coronaria, el $52.6 \%$ enfermedad renal crónica, el $49.1 \%$ hipotiroidismo o hipertiroidismo, $45.6 \%$ EPOC, $43 \%$ hipertensión pulmonar y $29.8 \%$ diabetes mellitus tipo 2 .

La mayoría de los pacientes se encontraban en una clase funcional NYHA III (48.2\%) y IV (41.2\%) y $80.6 \%$ ingresaba en Stevenson B, documentándose congestión pulmonar en $49.1 \%$ de los casos; en estadio C de la AHA el $81.6 \%$ y Del 14.9\%; la presión arterial sistólica promedio de ingreso fue $131 \mathrm{mmHg}$ y $76.6 \%$ de los pacientes tenia frecuencia cardiaca mayor de $70 \mathrm{lpm}$.

\section{Inicio de la insuficiencia cardiaca}

El $86.8 \%$ correspondía a pacientes con insuficiencia cardiaca conocida que habían desarrollado una descompensación y $7.9 \%$ se presentaban en una forma aguda de novo.

\section{Características bioquímica sanguínea}

Se encontró que el promedio de hemoglobina fue de 11.6 $\mathrm{g} / \mathrm{dL}( \pm 2.51)$, correspondiente a rango de anemia, con hematocrito $35.7( \pm 7.45)$, los hallazgos de electrolitos fueron compatibles con hiponatremia con una mediana de sodio de $133.5 \mathrm{mEq} / \mathrm{L}$ (rango IQ 6.25), potasio normal $4.77 \mathrm{mEq} / \mathrm{L}$ (rango IQ 0.75), sin embargo el promedio de BUN fue de $44.2 \mathrm{mg} / \mathrm{dL}$ (DS 22.5) con una mediana de creatinina 1.54 $\mathrm{mg} / \mathrm{dL}$ (rango IQ 0.87) compatibles con insuficiencia renal; la mediana de lactato también se encontró elevada en 2.6 $\mathrm{mg} / \mathrm{dL}(1,8)$, la TSH fue medida en 53\% de los pacientes, de ellos $58 \%$ se encontraron valores mayores al límite superior de la normalidad; no se encontraron datos suficientes

Tabla 2. Características bioquímica sanguínea.

\begin{tabular}{|c|c|c|}
\hline & Media/Mediana* & DS/Rango IQ* \\
\hline \multicolumn{3}{|l|}{ Bioquímica } \\
\hline Hemoglobina g/dL & 11.6 & 2.52 \\
\hline Hematocrito \% & 35.77 & 7.45 \\
\hline RDW\%* & 16.20 & 3.13 \\
\hline Sodio $\mathrm{mEq} / \mathrm{L}^{*}$ & 133.50 & 6.25 \\
\hline Potasio $\mathrm{mEq} / \mathrm{L}^{*}$ & 4.77 & 0.75 \\
\hline Troponina $\mathrm{ng} / \mathrm{mL} *$ & 0.04 & 0.05 \\
\hline BUN mg/dL & 44.20 & 22.50 \\
\hline Creatinina mg/dL & 1.55 & 0.87 \\
\hline Lactato $\mathrm{mg} / \mathrm{dL} *(\mathrm{n}=84)$ & 2.6 & 1.8 \\
\hline $\mathrm{TSH} \mathrm{mUI} / \mathrm{L}^{*}(\mathrm{n}=61)$ & 5.2 & 8.5 \\
\hline Linfocitos $/ \mathrm{mm}^{3}$ & 1957.02 & 654.0 \\
\hline Plaquetas $/ \mathrm{mm}^{3}$ & 226517.54 & 111132.4 \\
\hline Magnesio mEq/L ( $\mathrm{n}=85)$ & 1.81 & 0.3 \\
\hline Glicemia mg/dL $(\mathrm{n}=79)$ & 100.45 & 29.7 \\
\hline
\end{tabular}


de ferritina, saturación de transferrina, bilirrubinas, perfil lipídico, ácido úrico, ni de péptidos natriuréticos (Tabla 2).

\section{Características ecocardiográficas Ventrículo izquierdo}

En general, el promedio de los pacientes tenían diámetros del ventrículo izquierdo en rango de dilatación, los volúmenes fueron reportados en $60 \%$ de los ecocardiogramas revisados; el SLG fue reportado solamente en $19 \%$ de los casos; el volumen auricular izquierdo fue reportado en $90 \%$ de los casos con un promedio de 54.3 $\mathrm{mL} / \mathrm{m}^{2},( \pm 22.3)$; en $71.9 \%$ de los pacientes se encontró algún grado de disfunción diastólica; la insuficiencia mitral fue reportada en $83.3 \%$ de los casos, en su mayoría leve

Tabla 3. Características ecocardiográficas.

\begin{tabular}{|c|c|c|}
\hline & Media/Mediana* & DS/Rango IQ* \\
\hline \multicolumn{3}{|l|}{ Ecocardiografía } \\
\hline DTD cm & 5.53 & 0.90 \\
\hline DTS cm & 4.64 & 1.04 \\
\hline SLG $\% *(n=22)$ & -7.00 & -3.00 \\
\hline $\mathrm{VAI} \mathrm{mL} / \mathrm{m} 2$ & 54.34 & 22.30 \\
\hline PSAP*mmHg $(n=102)$ & 50.00 & 17.30 \\
\hline \multirow[t]{2}{*}{ TAPSE* ${ }^{*} \mathrm{~cm}(\mathrm{n}=83)$} & 1.70 & 0.60 \\
\hline & $\mathbf{n}$ & $\%$ \\
\hline \multicolumn{3}{|l|}{ Disfunción diastólica } \\
\hline Tipo I & 30 & 26.3 \\
\hline Tipo II & 23 & 20.2 \\
\hline Tipo III & 29 & 25.4 \\
\hline Ausencia & 32 & 28.1 \\
\hline \multicolumn{3}{|l|}{ Insuficiencia mitral } \\
\hline Leve & 54 & 47.4 \\
\hline Moderada & 29 & 25.4 \\
\hline Severa & 12 & 10.5 \\
\hline Ausencia & 19 & 16.7 \\
\hline \multicolumn{3}{|l|}{ Insuficiencia aórtica } \\
\hline Leve & 50 & 43.9 \\
\hline Moderada & 16 & 14 \\
\hline Severa & 4 & 3.5 \\
\hline Ausencia & 44 & 38.6 \\
\hline \multicolumn{3}{|l|}{ Insuficiencia tricuspídea } \\
\hline Leve & 65 & 57 \\
\hline Moderada & 26 & 22.8 \\
\hline Severa & 6 & 5.3 \\
\hline Ausencia & 17 & 14.9 \\
\hline $\begin{array}{l}\text { DTD: diámetro teledias } \\
\text { tudinal global, VAI: vol } \\
\text { arteria pulmonar, }\end{array}$ & $\begin{array}{l}\text { S: diámetro te } \\
\text { cular izquierdo, }\end{array}$ & $\begin{array}{l}G: \text { strain long } \\
\text { ón sistólica de }\end{array}$ \\
\hline
\end{tabular}

(47.4\%); la insuficiencia aórtica se encontró en $61.4 \%$ de los casos siendo leve en $43.9 \%$ (Tabla 3 ).

\section{Ventrículo derecho}

El $85.1 \%$ de los pacientes tenía algún grado de insuficiencia tricuspídea, siendo leve en la mayoría de los casos; se encontró una mediana de PSAP de $50 \mathrm{mmHg}$; del 72.8\% de los pacientes que tenían reporte de TAPSE en el ecocardiograma, se encontró una mediana de $17 \mathrm{~mm}$; no se encontraron datos suficientes acerca de área de cambio fraccional, ni velocidad sistólica del anillo tricuspídeo.

\section{Tratamiento farmacológico}

El $71.1 \%$ de los pacientes recibían tratamiento con IECAS o ARA-II, sólo $10.5 \%$ de los pacientes tomaban dosis óptimas de IECA; el $88.6 \%$ recibían betabloqueantes, en su mayoría carvedilol (70.2\%); sin embargo, sólo 3.5\% se encontraba en dosis óptimas; el segundo betabloqueador más usado fue metoprolol tartrato y sólo $7 \%$ en dosis óptimas; el $57.6 \%$ de los pacientes recibían antagonistas de aldosterona ( $100 \%$ con espironolactona), de los cuales $1.7 \%$ se encontraba en dosis óptimas; a $71.9 \%$ de los pacientes se les dio egreso con diuréticos de asa en una dosis de $40 \mathrm{mg} /$ día, $16.7 \%$ de los pacientes recibieron digoxina, $60.5 \%$ estatinas y $56.1 \%$ antiagregantes, $7.9 \%$ de los pacientes recibieron dosis de levosimendan durante su estancia hospitalaria (Tabla 4).

Con respecto al uso de dispositivos el $45.6 \%$ tenían CDI, el $31.6 \%$ marcapaso y el $22.8 \%$ terapia de resincronización cardiaca.

La mediana de estancia hospitalaria fue de nueve días (rango IQ de 20 días); del total de pacientes revisados 25.4\% requirió manejo en UCI con un promedio de 6.6 (DS 5.08) días; se reportó una mortalidad intrahospitalaria de $18 \%$. El $75 \%$ de los pacientes correspondía a fuerzas militares (ejército).

En su mayoría la especialidad tratante de los pacientes hospitalizados fue medicina interna $(73 \%)$ y en segundo lugar cardiología (27\%).

Se realizó optimización ambulatoria en $32 \%$ de los pacientes y en el $68 \%$ restante no se encontró titulación de medicamentos en controles ambulatorios.

El $92 \%$ de los pacientes que consultaron a urgencias requirió hospitalización, y el $8 \%$ restante corresponde a pacientes que consultaron a urgencias con tiempo de observación requerido inferior a un día dándose alta; el 38\% de los 114 pacientes reingresaron al menos una vez. De ellos, la mayoría $(68 \%)$ reingresó dentro los primeros 30 días. La mortalidad para reingresos tempranos fue 16 y $3 \%$ para pacientes con ingresos tardíos (Tabla 5).

En el análisis bivariado para el desenlace de mortalidad hospitalaria, las variables que tuvieron significancia estadística fueron: estadio AHA D (OR=3.68, IC 95\% 1.14$11.8, \mathrm{p}=0.028)$ estatinas $(\mathrm{OR}=0.34$, IC $95 \%$ 0.12-0.95, $\mathrm{p}=0.035), \operatorname{ASA}(\mathrm{OR}=0.17$, IC 95\% 0.046-0.65, $\mathrm{p}=0.035)$ 
Tabla 4. Tratamiento farmacológico.

\begin{tabular}{|c|c|c|}
\hline Tratamiento previo al egreso & $\mathbf{n}$ & $\%$ \\
\hline ARA II & 40 & 35.1 \\
\hline IECA & 41 & 36 \\
\hline $2.5 \mathrm{mg}$ BID & 10 & 8.8 \\
\hline $5 \mathrm{mg}$ BID & 18 & 15.8 \\
\hline $10 \mathrm{mg}$ BID & 1 & 0.87 \\
\hline $20 \mathrm{mg}$ BID & 12 & 10.5 \\
\hline Betabloqueador & 101 & 88.6 \\
\hline Carvedilol & 80 & 70.2 \\
\hline $6.25 \mathrm{mg}$ BID & 44 & 38.6 \\
\hline $12.5 \mathrm{mg}$ BID & 17 & 14.9 \\
\hline 25 mg BID & 4 & 3.5 \\
\hline $3.12 \mathrm{mg}$ BID & 15 & 13.1 \\
\hline Metoprolol tartrato & 19 & 16.6 \\
\hline 25 mg BID & 5 & 4.4 \\
\hline $50 \mathrm{mg}$ BID & 8 & 7 \\
\hline Antagonistas de Aldosterona & 66 & 57.6 \\
\hline 25 mg día & 47 & 41.2 \\
\hline 50 mg día & 2 & 1.7 \\
\hline 12.5 mg día & 15 & 13.1 \\
\hline \multicolumn{3}{|l|}{ Furosemida } \\
\hline 40 mg día & 82 & 71.9 \\
\hline Digital & 19 & 16.7 \\
\hline $\mathrm{ACO} / \mathrm{SC}$ & 25 & 21.9 \\
\hline Estatinas & 69 & 60.5 \\
\hline Nitratos & 6 & 5.3 \\
\hline Amiodarona & 25 & 21.9 \\
\hline Levosimendan & 9 & 7.9 \\
\hline Antiagregante & 64 & 56.1 \\
\hline $\mathrm{ACO} / \mathrm{SC}$ : anticoagulantes or & & \\
\hline
\end{tabular}

betabloqueadores $(\mathrm{OR}=0.23$, IC 95\% 0.67-0.83, $\mathrm{p}=0.033)$, los cuales se presentan en la Tabla 5.

\section{Discusión}

Este es el primer estudio de pacientes hospitalizados con insuficiencia cardiaca descompensada con fracción de eyección reducida, realizado en el Hospital Militar Central. El análisis descriptivo permite caracterizar a los pacientes con esta patología, con el fin de establecer los determinantes que llevan a la descompensación de la insuficiencia cardiaca y proponer alternativas de orientación terapéutica que impacten en la atención brindada a nuestra población, teniendo en cuenta que se trata de una de las principales causas de consulta al servicio de urgencias y hospitalización; así mismo, a pesar de tratarse de un estudio descriptivo se realizó una exploración de las posibles variables relacionadas con mortalidad.
Tabla 5. Reingresos.

\begin{tabular}{|l|c|c|}
\hline & N/ Mediana* & \%/Rango IQ* \\
\hline Total pacientes con reingresos & $\mathbf{3 1}$ & $\mathbf{3 8}$ \\
Reingresos tempranos (menor 30 días) & 21 & 68 \\
Reingresos tardíos (31-90 días) & 10 & 32 \\
\hline Mortalidad total reingresos & $\mathbf{6}$ & $\mathbf{1 9}$ \\
Mortalidad reingresos tempranos (1-30 días) & 5 & 16 \\
Mortalidad reingresos tardío (31-90 días) & 1 & 3 \\
Número reingresos* & 1 & 89 \\
Días hasta reingreso* & 20 & 1 \\
\hline
\end{tabular}

El promedio de edad fue 74.8 años, hubo alta proporción de comorbilidades como HTA, enfermedad coronaria, enfermedad renal crónica, enfermedad tiroidea, EPOC e hipertensión pulmonar; con respecto a la etiología de la insuficiencia cardiaca, la HTA y la enfermedad isquémica se encontraron entre las dos causas más frecuentes, datos similares a lo reportado en Colombia y Latinoamérica (11, 12); sin embargo, la diferencia de estos estudios y otros reportados en la literatura mundial (13), predominó el sexo masculino, hallazgos que son similares al registro chileno de insuficiencia cardiaca en donde $73.7 \%$ correspondían al género masculino, y en el nuestro $69.3 \%$; sin embargo, en el presente trabajo puede influenciar el hecho de ser en su mayoría hombres los adscritos a las fuerzas militares.

En cuanto a los factores precipitantes de la insuficiencia cardiaca, la no adherencia al tratamiento sigue siendo una de las principales causas de descompensación lo cual plantea la importancia de la educación de estos pacientes y de la evaluación de su red de apoyo social para fortalecer las medidas de adherencia al tratamiento farmacológico y no farmacológico; sin embargo, se ha descrito previamente la no adherencia como un factor asociado con un tiempo de estancia hospitalaria no prolongado (14); en segundo lugar la etiología infecciosa y en tercero el síndrome coronario agudo como causas más frecuentes de descompensación fueron descritas en nuestro estudio

La mayoría tienen insuficiencia cardiaca crónica conocida que presentaron una descompensación intercurrente.

En un importante porcentaje de los pacientes se registró congestión pulmonar al momento del ingreso (49.1\%), a este respecto, es importante su detección y tratamiento intensivo durante la estancia en el servicio de urgencias y/o hospitalización, pues existe evidencia de que la persistencia de la congestión al alta hospitalaria, provoca un aumento de riesgo de muerte y de rehospitalizaciones por insuficiencia cardiaca (15).

En cuanto a la severidad de la descompensación al ingreso se registró un alto porcentaje de pacientes con clase funcional NYHA III - IV (89.4\%), esto puede ser un factor 
que explique en parte la mayor estancia hospitalaria vista en el presente estudio, lo cual también podría influir en la mortalidad y la consecuente realización de un gran número de exámenes complementarios (hemograma, bioquímica, coagulación, gasometría, radiografía de tórax, electrocardiograma, enzimas cardiacas), además en falla cardiaca aguda la elevación de la troponina es multifactorial (sepsis, arritmias, anemia) y en gran parte de los casos reversible, por lo que aún no se ha definido su utilidad como toma única o seriada en estos pacientes (16).

Cabe destacar la ausencia de medición de biomarcadores de insuficiencia cardiaca como el BNP o NT-pro-BNP, a pesar de que han demostrado ser un marcador con alta sensibilidad para el diagnóstico de insuficiencia cardiaca aguda, y un importante predictor pronóstico y de respuesta al manejo, que además ayuda a decidir el ingreso del paciente, la realización de ecocardiograma y a orientar decisiones terapéuticas (17-18); es prioritario que en un futuro próximo esta situación cambie y estos biomarcadores pasen a formar parte de la práctica clínica diaria en el HMC.

Los hallazgos ecocardiográficos son esperables para una población con falla cardiaca con fracción de eyección reducida; sin embargo, es importante la adherencia a las guías de cuantificación de cámaras en cuanto a la valoración de volúmenes del ventrículo izquierdo, para, en un futuro establecer estudios comparativos que identifiquen criterios de remodelado reverso asociados al manejo farmacológico, la educación y la rehabilitación cardiaca, igualmente estimular estudios que incluyan el strain como un marcador etiológico y pronóstico en este grupo de pacientes así como la valoración completa de la función ventricular derecha y el tamizaje de hipertensión pulmonar con resistencias pulmonares elevadas importante en orientaciones diagnósticas y terapéuticas en este grupo de pacientes.

Es de destacar que el tratamiento farmacológico basados en la evidencia y recomendado por las guías de práctica clínica al momento del egreso hospitalario, fue formulado en un alto porcentaje de los casos, así, un IECA o un ARAII fue formulado en $71.1 \%$ de los casos, un betabloqueador en $88.6 \%$ y un antagonista de la aldosterona en $57.6 \%$; sin embargo, en un muy bajo porcentaje de los pacientes se dio egreso con dosis óptimas recomendadas (10.5\% para IECA (enalapril), $3.5 \%$ para betabloqueadores (carvedilol) y $1.7 \%$ para AA (espironolactona), lo cual refleja la necesidad de implantar estrategias de prescripción ante su demostrado beneficio en la morbimortalidad de estos pacientes (19-21).

Se ha descrito mayor adherencia a recomendaciones de guías de manejo, cuando el servicio tratante es cardiología si bien para el presente estudio no existe muestra suficiente que permita determinar diferencias según servicio tratante y de esta manera mejorar los procesos de atención $(22,23)$.

Si tenemos en cuenta que los pacientes analizados poseen una disfunción sistólica y como vimos en su mayoría consultan con hipoperfusión periférica (NYHA III-IV, congestión pulmonar, disminución de la función renal, lactato elevado) sumado a que probablemente muchos de ellos son refractarios a diuréticos y/o vasodilatadores, parece razonable esperar una mayor utilización de fármacos como el levosimendán dados sus beneficios clínicos y hemodinámicos demostrados (24-26), no obstante, el grado de severidad de descompensación determinado (en su mayoría Stevenson B), también podría explica la baja frecuencia en el uso de inotropia (27).

La optimización farmacológica ambulatoria determinada debe analizarse, de acuerdo con la posibilidad de que los pacientes hospitalizados por descompensación reciban titulación farmacológica cada dos semanas a partir del egreso, modelo que ha probado costo-eficacia en las clínicas ambulatorias de falla cardiaca.

La no disponibilidad de información completa acerca de los hallazgos electrocardiográficos junto con el alto porcentaje de pacientes con dosis subóptimas de tratamiento farmacológico no permiten determinar si todos los pacientes que tendrían indicación cuentan con terapia de resincronización cardiaca, adicional al hecho de que el escenario de estudio fuese intrahospitalario.

Si bien, de aumentarse el número de pacientes que reciben terapia médica óptima, se podría determinar, qué porcentaje de población realmente tendría la indicación y el beneficio de una resincronización cardiaca. En el caso de los desfibriladores, la titulación a dosis máximas sustentadas en ensayos clínicos, podría llevar a la disminución de las descargas apopiadas $(28,29)$.

Por otro lado, la mediana de estancia hospitalaria estuvo dentro del número de días promedio reportado en otros estudios; sin embargo, el rango intercuartílico reportado en el presente estudio refleja la dispersión de los datos obtenidos.

Aunque, la mayoría de pacientes que consultan a urgencias requieren hospitalización, lo cual está en relación con el grado de descompensación Stevenson, siendo B, el más frecuente, se considera que un manejo farmacológico de descongestión o titulación de medicamentos de acuerdo con guías, permitiría reducir hospitalizaciones y además, manteniendo este proceso de titulación en forma ambulatoria (30).

La mortalidad intrahospitalaria resultó mayor al promedio de países latinoamericanos (Brasil 8.5\%, México $2.9 \%$ ) como para Norteamérica (2.7\%) (31); sin embargo, estos registros de mortalidad en su mayoría son analizados teniendo como unidad de análisis las hospitalizaciones y no el desenlace individual por paciente.

El porcentaje de reingresos presentado se encuentra en relación con los calculados en varios registros $(32,33)$ que determinan readmisiones de hasta $25 \%$ de los pacientes dentro de los primeros 30 días con mortalidad cercana al $10 \%$ en este periodo. Sin embargo, se resalta el alto número de reingresos (38\%) presentándose dentro de la denominada "fase vulnerable" correspondiente a los primeros 30 días posteriores al egreso. Este periodo se constituye en aquel con alta mortalidad prevenible mediante la evaluación ambulatoria precoz idealmente en los primeros siete días del 
egreso, para fortalecer las conductas educativas preventivas de autocuidado junto con la titulación hasta dosis médicas óptimas o máximas toleradas de los fármacos que han mostrado disminución en morbimortalidad de los pacientes con falla cardiaca.

Entendiendo las limitaciones propias del estudio, los resultados de la exploración mediante el análisis bivariado de posibles factores de asociación de la antiagregación con ASA, uso de estatinas y betabloqueadores podrían explicarse por un porcentaje más alto de pacientes con cardiopatía isquémica $(60.5 \%)$ como una de las etiologías más frecuentes de la falla cardiaca en la presente descripción.

Una vez conocidas las características de la población con falla cardiaca con fracción de eyección reducida, las causas más frecuentes de descompensación, comorbilidades, perfil clínico y el alto porcentaje de reingresos, resulta en un interesante blanco de intervenciones activas en forma temprana durante la hospitalización y la evaluación ambulatoria precoz a través de un equipo multidisciplinario enmarcado dentro del modelo de atención propuesto por las clínicas de falla cardiaca. Estos programas han demostrado ampliamente sus beneficios y son amparados por las guías de práctica clínica tanto nacionales como internacionales.

\section{Limitaciones del estudio}

Se trata de un estudio descriptivo desarrollado durante el año 2015 para una población fija, debido a su afiliación como miembros de las fuerzas militares y sus familias siendo por tanto un estudio de un solo centro de atención. Sin embargo al ser el centro de referencia nacional puede contar con la diversidad de población representativa del país y permite hacer el seguimiento de los pacientes que por descompensaciones sólo consultarían a la red de atención en salud de las fuerzas militares. El no contar con medición de péptidos natriuréticos limita la capacidad de exclusión diagnóstica de los pacientes con falla cardiaca en comparación a otros estudios, aunque la totalidad de los pacientes incluidos contaban con datos ecocardiográficos que respaldaran el diagnóstico.

El tamaño de muestra podría limitar la determinación de factores de asociación si bien no es parte del propósito del mismo al ser un estudio descriptivo.

\section{Conclusiones}

El presente estudio permitió describir las principales características de los pacientes con insuficiencia cardiaca con fracción de eyección reducida descompensada que son admitidos a un hospital de cuarto nivel. El perfil clínico de los pacientes es semejante a los reportados en diferentes series, sin embargo el género masculino predominó en la admisión. La adherencia a guías de manejo al momento del egreso es adecuada pero en dosis subóptimas debiendo intervenirse la fase inmediata al alta dado el alto porcentaje de reingreso registrados así como la mortalidad intrahospitalaria reportada. Estos hallazgos plantean la necesidad de crear una clínica de falla cardiaca para el manejo ambulatorio permitiendo disminuir el número de ingresos, reingresos, días de hospitalización, junto con un acceso fácil a los servicios y seguimiento oportuno de los pacientes.

Se requiere ampliar el tamaño del registro con miras a un análisis de factores de asociación, desenlaces y construcción del conocimiento.

\section{Agradecimientos}

Al Hospital Militar Central y los Cardiólogos Juan Pérez Rapalino y Margarita Blanco por su apoyo en este estudio."

\section{Referencias}

1. Mcmurray JJV, Adamopoulos S, Anker SD, Auricchio A, Böhm M, Dickstein $\mathbf{K}$, et al. ESC Guidelines for the diagnosis and treat- ment of acute and chronic heart failure 2012. Eur J Heart Fail. 2012;14(8):803-69.

2. Yancy CW, Jessup M, Bozkurt B, Butler J, Casey DE, Drazner MH, et al 2013 ACCF/AHA guideline for the management of heart failure: a report of the American College of Cardiology Foundation/American Heart Association Task Force on Practice Guidelines. J Am Coll Cardiol. 2013;62(16):e147-239.

3. Hernández-Leiva E. Epidemiologia del síndrome coronario agudo y la insuficiencia cardiaca en Latinoamérica. Rev Esp Cardiol. 2011;64 Supl. 2:34-43.

4. SISPRO. Bodega de datos de SISPRO (RIPS) - Ministerio de Salud y Protección Social. Reporte: prestaciones de servicios de salud reportados por las entidades administradoras de planes de beneficio para los años 2009, 2010 y 2011, según resolución 3374/00. Actualización a 31-01-2013. Consultado: 28-04-2014.

5. Senior J, Saldarriaga C, Rendón J. Descripcion clínico - epidemiológica de los pacientes con falla cardiaca aguda que consultan al servicio de urgencias. Acta Med Colomb. 2011;36:125-9.

6. Tamayo D, Rodríguez V, Rojas M, Rincón M, Franco C, Ibarra M, et al. Costos ambulatorios y hospitalarios de la falla cardiaca en dos hospitales de Bogotá. Acta Med Colomb. 2013;38:208-12.

7. American College of Cardiology Foundation/American Heart Association. (2013). 2013 ACCF/AHA guideline for the management of heart failure: Executive summary: A report of the American College of Cardiology Foundation/ American Heart Association task force on practice guidelines. Circulation, 128, 1810-1852.

8. Lainscak, M. (2004). Implementation of guidelines for management of heart failure in heart failure clinic: Effects beyond pharmacological treatment. International Journal of Cardiology, 97, 411-416. doi:10.1016/j.icard.2003.10.031.

9. Mant, J., Al-Mohammad, A., Swan, S., \& Laramee, P. (2011). Management of chronic heart failure in adults: Synopsis of the national institute for health and clinical excellence guideline. American College of Physicians, 155(4), 252-260. www.annals.org.

10. Whitlock, A. M. (2010). Acute heart failure: Patient assessment and management. British Journal of Cardiac Nursing, 5(11), 516-526

11. Gómez, Efrain. Introducción, epidemiología de la falla cardiaca e historia de las clínicas de falla cardiaca en Colombia Rev Colomb Cardiol. 2016;23(S1):6-12

12. Edimar Alcides Bocchi, MD, Alexandra Arias, MD, Hugo Verdejo, MD, Mirta Diez, MD, Efraín Gómez, MD, Pablo Castro, MD, The Reality of Heart Failure in Latin America, 2013 by the American College of Cardiology Foundation, Vol. 62, No. 11, 2013

13. Fonarow GC, Abraham WT, Albert NM, et al. Organized Program to Initiate Lifesaving Treatment in Hospitalized Patients with Heart Failure (OPTIMIZEHF): rationale and design. Am Heart J 2004; 148(1): 43-51.

14. Ambardekar AV, Fonarow GC, Hernandez AF, et al. Characteristics and in- hospital outcomes for nonadherent patients with heart failure: findings from Get With The Guidelines-Heart Failure (GWTG-HF). Am Heart J. 2009;158: 644-52.

15. Gheorghiade M, Filippatos G, de Luca L, Burnett J. Conges- tion in acute heart failure syndromes: an essential target of evaluation and treatment. Am J Med 2006;119:S3-S10.

16. Masip J. No-invasive ventilation. Heart Fail Rev 2007;12:119-24. Januzzi J, Van Kimmenade R, Lainchbury J, Bayes-Genis A, Ordonez-Llanos J, Santalo M, et al. NT-proBNP testing for diagnosis and short-term prognosis in acute destabilized heart failure: an international pooled analysis of 1256 patients. Eur Heart J 2006;27:330-7.

17. Jourdain P, Jondeau G, Funck F, Gueffet P, Le Helleco A, Donal E, et al. Plasma brain natriuretic peptide-guided therapy to improve outcome in heart failure. J Am Coll Cardiol 2007;49:1733-9.

18. Almenar-Bonet L, Martínez-Dolz L. Péptidos natriuréticos en insuficiencia 
cardiaca. Rev Esp Cardiol 2006;15F-26F.

19.Siirilä-Waris K, Lassus J, Melón J, Peuhkurinen K, Nieminem M, Harjola $\mathbf{V}$ et al. Characteritics, outcomes, and predictors of 1-yeart mortality in patients hospitalized for acute heart failure. Eur Heart J 2006;27:3011-7.

20.O'Connor CM, Durbam NC. The new heart failure guideli- nes: strategies for implementation. Am Heart J 2007;153:S2-S5.

21. Metra M, Torp-Pedersen, Cleland J, Lenarda AD, Komajda M, Remme WJ, et al. Should betablocker therapy be reduced or withdrawn after an episode of descompensated heart failure? Results from COMET. Eur J Heart Fail 2007; doi:10.1016/j.ejheart.2007.05.011.

22.Philbin EF, Jenkins PL. Differences between patients with heart failure treated by cardiologists, internists, family physicians, and other physicians: analysis of a large, statewide database. Am Heart J. 2000 Mar;139(3):491-6.

23. Stawicki S, Roik M, Jasik M, Kosior D, Scisło P, Kochanowski J, Dembe K, Steckiewicz R, Karnafel W, Opolski G. Differences between cardiologists and internists in the management of heart failure. Medical guidelines compared with every-day practice. Kardiol Pàol. 2004 Nov;61(11):431-9; discussion 440-1.

24.Llorens P, Carbajosa J, Fernandez-Cañadas J, Murcia J, Climent V, Laghzaoui F, et al. Experiencia clínica con levosi- mendán en un servicio de urgencias de un hospital de ter- cer nivel. Rev Esp Cardiol 2007;60:543-47.

25.Parissis JT, Farmakis D, Niemine M. Classical inotropes and new cardiac enhancers. Heart Fail Rev 2007;12:149-56.

26. De Luca L, Coluucci W, Nieminen M, Massie B, Gheorghiade M. Evidence-based use of levosimendan in different clinical-settings. Eur Hear J 2006;27:1908-20
27. Nieminen M, Brutsaert D, Dickstein K, Drexler H, Follath F, Harjola V, et al. Euroheart Failure Survey-II (EHFS II): a survey on hospitalized acute heart failure patients: description of population. Eur Heart $J$ 2006;27:2725-36.

28. Ruwald MH, Abu-Zeitone A, Jons C, Ruwald AC, McNitt S,Kutyifa $\mathbf{V}$, et al. Impact of carvedilol and metoprolol on inappropriate implantable cardioverter-defibrillator therapy: theMADIT-CRT trial (Multicenter Automatic Defibrillator Implantation With Cardiac Resynchronization Therapy. J Am Coll Cardiol.2013;62(15):1343-50.

29.Schmidt S, Hürlimann D, Starck CT, Hindricks G, LüscherTF, Ruschitzka F, et al. Treatment with higher dosages ofheart failure medication is associated with improved out-come following cardiac resynchronization therapy. Eur Heart $J .2014 ; 35(16): 1051-60$.

30. West R, Liang L, Fonarow G, et al. Characterization of heart failure patients with preserved ejection fraction: a comparison between ADHERE-US registry and ADHERE-International registry. Eur J Heart Fail 2011;13:945-52

31. Bocchi EA, Arias A, Verdejo H, Diez M, Gómez E, Castro P. The reality of heart failure in Latin America. J Am Coll Cardiol. 2013 Sep 10;62(11):949-58. doi: 10.1016/j.jacc.2013.06.013. Epub 2013 Jul 10.

32.Dharmarajan, K. et al. Diagnoses and timing of 30-day readmissions after hospitalization for heart failure, acute myocardial infarction, or pneumonia. JAMA 309, 355-363 (2013).

33. Bueno, H. et al. Trends in length of stay and short-term outcomes among Medicare patients hospitalized for heart failure, 1993-2006. JAMA 303, 2141-2147 (2010) 